\title{
PAPEL DEL VOLUMEN PROSTÁTICO EN LA BIOPSIA TRANSRECTAL ECODIRIGIDA DE PRÓSTATA: ¿ES UN MARCADOR TAN IMPORTANTE COMO EL PSA?
}

\author{
Rafael Rodríguez-Patrón Rodríguez, Teodoro M a yayo Dehesa, Francisco Javier Burgos Revilla, \\ Mónica Alonso González, Alberto Lennie Zucharino y Ricardo G arcía G onzález.
}

Unidad de Ecografía Urológica ${ }^{1}$. Universidad de Alcalá de Henares.

Técnico de Salud Area $8^{2}$. M adrid. España

\begin{abstract}
Resumen.- O BJETIVO: La limitación del PSA para identificar a los pacientes con cáncer prostático ha hecho que se definan diferentes parámetros que aumenten su especificidad sin reducir su sensibilidad de forma importante. En este trabajo se estudia la relación del volumen con la presencia de cáncer de próstata en biopsia sextante.

MÉTO DO : se han recogido los resultados de las biopsias prostáticas realizadas a 6000 pacientes entre 1994 y 2002. De ellos 861 se han biopsiado en más de una ocasión, sumando un total de 7127 biopsias. Se realizaron diferentes modelos predictivos para identificar los factores relacionados con la positividad de la biopsia.
\end{abstract}

RESULTADO S: La media de volumen prostático es de $40,6 \pm 66,2$ c.c. en la primera biopsia aumentando en las sucesivas biopsias hasta los 85,17 c.c. Se observa una alta incidencia de $C P$ en las próstatas pequeñas que alcanza el $67,2 \%$ en las de tamaño normal (menores de 20 c.c.) que disminuye a medida que aumenta el volumen encontrando únicamente un $19,7 \%$ en a quellas mayores de 50 c.c. $(p<0,0001)$. En pacientes con PSA entre 4 y $10 \mathrm{ng} / \mathrm{ml}$ y segundas biopsias el porcentaje de cáncer con glándulas mayores de $50 \mathrm{cc}$ fue inferior al 10\%. En la regresión logística multivariante mostraron relación con la positividad el PSA, el volumen y la Densidad de PSA pero no la relación PSA Libre/ PSA total.

CON CLUSIO NES: LOS puntos de corte estándar de PSA no son adecuados para un correcto diagnóstico de C P mediante BTE. El volumen (HPB) influye de manera determinante en sus cifras y en la rentabilidad de la prueba por lo que es preciso tenerlo en consideración al indicar la biopsia.

Palabras clave: Biopsia transrectal prostática. Cáncer prostático. Volumen prostático.

Summary.- O BJEC TIVES: The limitations of PSA to identify patients with prostate cancer prompted the definition of different parameters trying to increase specificity without reducing sensitivity. This paper studies the relationship of volume and presence of prostate cancer in sextant biopsies. 
METHO DS: W e collected the results of prostate biopsies performed to 6000 patients between 1994 and 2002 . 861 of them underwent more than one biopsy, adding up for a total of 7127 biopsies. Various predictive models to identify factors related to positive biopsy were constructed.

RESULTS: M ean prostate volume is $14.6 \pm 66.2 \mathrm{cc}$ for the first biopsy, increasing in successive biopsies to $85.17 \mathrm{cc}$. A high incidence of prostate cancer was observed in small prostates, reaching $67.2 \%$ of those with normal size $(<20 \mathrm{cC})$ and diminishing with the increase of volume down to only $19.7 \%$ in those larger than $50 \mathrm{CC}(p<0.0001)$. In second biopsies of patients with PSA between 4 and $10 \mathrm{ng} / \mathrm{ml}$ and gland volume higher than $50 \mathrm{cc}$ percentage of biopsies positive for cancer was below $10 \%$. Multiva riant logistic regression showed that PSA, volume and PSA density were related with positive biopsies, but not free/ total PSA ratio.

CO N C LUSIO N S: Standard PSA cutoffs are not adequate for a proper diagnosis of prostate cancer by ultrasound guided transrectal biopsy. Volume (BPH) has a significant influence in PSA values and results of the biopsy, so that it should be taken into consideration when indicating biopsies.

Keywords: Transrectal prostate biopsy. Prostate cancer. Prostate volume.

\section{INTRODUCCIÓN}

En el diagnóstico médico sería ideal poseer un marcador tumoral que tenga suficiente sensibilidad como para detectar una gran mayoría de cánceres en un estadio inicial de la enfermedad cuando aún es posible la curación con las menores secuelas posibles y con una especificidad suficiente que evite diagnósticos invasivos a la mayor cantidad posible de pacientes. La aparición del Antígeno Prostático Específico (PSA) parecía cumplir estas expectativas a pesar de que no era un marcador tumoral como tal sino de tejido prostático pero pronto se hicieron evidentes sus limitaciones al comprobar el gran solapamiento de los niveles existentes entre pacientes con cáncer prostático (CP) y aquellos con Hipertrofia Prostática Benigna (HPB). Morote y cols (1) encuentran en un tercio de pacientes con HBP no complicada cifras también superiores a $4 \mathrm{ng} / \mathrm{ml}$. Por otro lado, entre un 18 y un 32
$\%$ de los varones con cáncer demostrado por biopsia tuvieron un PSA menor de $4,1 \mathrm{ng} / \mathrm{ml}$ (2). Por este motivo se buscó identificar los pacientes de mayor riesgo de CP mediante ajustes del PSA total en suero en base al volumen prostático (Densidad de PSA), sus formas en suero (relación PSA libre/ PSA total), la edad 0 la variación en el tiempo (velocidad de PSA). La asociación con el volumen prostático fue evidente desde muy temprano y en base a esta, Benson y cols. (3) describieron la Densidad de PSA (DPSA) o Babaian y cols (4) establecían rangos de PSA en función del volumen prostático.

En este trabajo se describen los resultados obtenidos acerca de la relación del volumen con la positividad de la biopsia sobre una serie de 6000 pacientes biopsiados.

\section{MATERIAL Y MÉTODO}

Se han recogido los resultados de 6000 pacientes biopsiados por vía transrectal entre 1994 y 2002. De los 6000 pacientes 674 han sido biopsiados 2 veces, 128 tres veces, 43 cuatro veces, 13 en cinco ocasiones, 2 en seis y 1 a un paciente se le practicaron 7 biopsias. En conjunto suman 7127 biopsias. Sólo se han incluido aquellos en los que se disponía del resultado de anatomía patológica y no tenían diagnóstico previo de malignidad. Salvo casos excepcionales (próstatas evidentemente tumorales en estadios avanzados, pacientes en mala situación general) se realizó biopsia sextante obteniendo las muestras de la zona periférica más lateral de la próstata.

Las biopsias se realizaron con diversos equipos de la serie Sonoline de Siemens (Siemens M edical Systems, Inc. Ultrasound G roup, Issaquah, WA). Para la obtención de muestras se utilizan agujas 18 Gauge tipo Tru-Cut modificadas para ser disparadas mediante el dispositivo Biopty-Bard ${ }^{\circ}$ (Bard urological Inc., A tlanta, Georgia), que obtienen cilindros de tejido de $1,5-2 \mathrm{~cm}$ de longitud con un diámetro de aproximadamente $1 \mathrm{~mm}$.

El cálculo del volumen se realizó después de obtener los diámetros anteroposterior y transversal según la fórmula $v=$ (Diámetro anteroposterior ${ }^{2} \mathrm{x}$ Diámetro Transversal)/2. Esta fórmula mantiene una correlación del 0,88 con el volumen real según Terris y cols (5) pero se ha mantenido desde el inicio por su 
sencillez de aplicación y para mantener resultados comparables con anteriores trabajos.

El análisis descriptivo incluye los porcentajes y percentiles para variables cualitativas y medidas de tendencia central para las variables cuantitativas continuas. En algunos casos las variables numéricas continuas se han categorizado de acuerdo a puntos de corte habituales en la práctica clínica y en la literatura.

Se determinaron varios modelos predictivos mediante regresión logística de las variables relacionadas con la presencia o no de cáncer. La selección de las variables para el modelo se basó en las que fueron significativas en el análisis bivariante estudiando si debían considerarse otras variables de importancia clínica. Se seleccionaron PSA, DPSA y volumen fraccionados en categorías, el TR y la presencia de una ecografía sospechosa (nódulos hipoecoicos o próstata heterogénea). Para no reducir excesivamente el número de pacientes el PSA L/T se incluyó en un modelo de regresión aparte que incorporaba además las mismas categorías de DPSA y volumen, tacto y ecografía. El análisis se realizó mediante un paquete estadístico SPSS 11.0 y SAS Software Release 8.2.

\section{RESULTADOS}

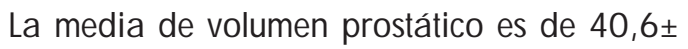
66,2 c.c. en la primera biopsia aumentando en las sucesivas biopsias hasta los 85,17 c.c. del paciente con siete biopsias. La Tabla I muestra los descriptivos en cada biopsia. Salvo entre la cuarta y quinta biopsias la diferencia de las medias es significativa con $p<0,05$.

Puede observarse también diferencia significativa en el volumen medio de los pacientes con HBP y los de CP en la primera biopsia tanto en el grupo general como en los pacientes con PSA intermedio entre 4 y $10 \mathrm{ng} / \mathrm{ml}$ (Tablas II y III). En la segunda biopsia puede apreciarse la misma situación y aunque en la tercera y cuarta la diferencia no resulta significativa se comprueba como en las anteriores que el tamaño prostático es menor en las próstatas tumorales (Tabla II).

Los pacientes con AGF presentan unos volúmenes similares a aquellos con CP mientras que los que tuvieron PIN se encuentran en cifras intermedias más cercanas a las de HBP.

Se han establecido rangos de volumen prostático para estudiar la incidencia de cáncer en ellos. Estos rangos son los siguientes: próstatas menores de 20 c.c., de 20 a 30 c.C., de 30 a 40 c.C., de 40 a 50 c.c y mayores de 50 c.c. la Tabla IV y la Figura 1 recogen la incidencia de la anatomía patológica en los distintos grupos de volumen en la primera biopsia.

Se observa una alta incidencia de CP en las próstatas pequeñas que alcanza el $67,2 \%$ en las de tamaño normal (menores de 20 c.c.) que disminuye a

TABLA I. VALO RES DESCRIPTIVOS DEL VO LUMEN EN LAS DISTIN TAS BIO PSIAS.

\begin{tabular}{|c|c|c|c|c|c|c|}
\hline & $\mathrm{N}$ & Media & Mediana & DT & M ínimo & Máximo \\
\hline \multicolumn{7}{|c|}{ VOLUMEN (cc) } \\
\hline 1 abiopsia & 5972 & 40.57 & 34.68 & 66.23 & 2.06 & 483.70 \\
\hline 2 abiopsia & 822 & 47.16 & 43.34 & 22.50 & 6.64 & 177.32 \\
\hline 3aBiopsia & 184 & 47.21 & 43.03 & 21.55 & 9.26 & 126.15 \\
\hline 4 abiopsia & 58 & 48.25 & 44.25 & 24.98 & 12.96 & 172.06 \\
\hline 5 abiopsia & 16 & 51.65 & 52.47 & 22.47 & 17.24 & 91.94 \\
\hline 6 abiopsia & 3 & 67.32 & 57.11 & 24.50 & 49.59 & 95.29 \\
\hline 7 abiopsia & 1 & 85.17 & 85.17 & & 85.17 & 85.17 \\
\hline
\end{tabular}


TABLA II. DESCRIPTIVOS DE VO LUMEN POR GRUPOS DE AN ATO M ÍA PATO LÓ G ICA.

\begin{tabular}{|c|c|c|c|c|c|c|c|c|}
\hline & & N & Mediana & Media & DT & Mín. & Máx. & $\begin{array}{l}\text { Anova } \\
\text { intergr. }\end{array}$ \\
\hline VO LUM EN & HBP & 3388 & 39.71 & 46.45 & 85.71 & 3.53 & 483.7 & $P<0,0001$ \\
\hline c.C. & CAN CER & 2336 & 27.16 & 32.16 & 20.11 & 2.35 & 379.31 & \\
\hline \multirow[t]{3}{*}{ 1a biopsia } & PIN & 122 & 39.62 & 42.19 & 17.93 & 13.44 & 112.69 & \\
\hline & $A G F$ & 126 & 32.41 & 36.98 & 18.19 & 2.06 & 111.25 & \\
\hline & Total & 5972 & 34.68 & 40.57 & 66.23 & 2.06 & 483.7 & \\
\hline VO LUM EN & HBP & 603 & 47.72 & 50.42 & 21.69 & 6.64 & 148.84 & \\
\hline c.c. & CANCER & 167 & 30.49 & 37.43 & 20.93 & 7.40 & 131.40 & $P<0,0001$ \\
\hline \multirow[t]{3}{*}{ 2a biopsia } & PIN & 24 & 37.63 & 41.97 & 21.31 & 15.67 & 110.47 & \\
\hline & $A G F$ & 28 & 32.41 & 39.34 & 30.29 & 12.81 & 177.32 & \\
\hline & Total & 822 & 42.72 & 47.16 & 22.90 & 6.64 & 177.32 & \\
\hline VOLUM EN & HBP & 142 & 46.46 & 49.12 & 21.80 & 9.26 & 126.15 & N.S. \\
\hline C.C. & CAN CER & 31 & 34.98 & 38.3 & 16.62 & 9.92 & 73.00 & $P<0,06$ \\
\hline \multirow[t]{3}{*}{ 3a biopsia } & PIN & 5 & 41.15 & 49.58 & 29.32 & 23.12 & 99.83 & \\
\hline & $A G F$ & 6 & 38.83 & 46.04 & 25.35 & 26.57 & 94.10 & \\
\hline & Total & 184 & 42.86 & 47.21 & 21.55 & 9.26 & 126.15 & \\
\hline VOLUMEN & HBP & 45 & 47.20 & 50.33 & 25.42 & 12.96 & 172.06 & n.s. \\
\hline c.c. & CAN CER & 7 & 34.34 & 38.30 & 14.62 & 22.53 & 60.02 & 0.54 \\
\hline \multirow[t]{3}{*}{4 ㅇ biopsia } & PIN & 2 & 32.92 & 32.98 & 20.03 & 18.82 & 47.15 & \\
\hline & SOS & 4 & 37.06 & 49.90 & 36.88 & 21.50 & 103.97 & \\
\hline & Total & 58 & 44.25 & 48.25 & 24.98 & 12.96 & 172.06 & \\
\hline
\end{tabular}

medida que aumenta el volumen encontrando únicamente un $19,7 \%$ en aquellas mayores de 50 c.c. $(p<0,0001)$. Por el contrario el porcentaje de PIN y
A GF es bastante estable entre grupos oscilando entre 1,1 y el $2,8 \%$. Cuando se comparan las características ecográficas y de TR (Tabla V) se aprecia que a menor

TABLA III. DESCRIPTIVOS DE VO LUMEN EN PRIM ERA BIO PSIA SEG ÚN DE AN ATO M ÍA PATO LÓ GICA EN PACIEN TES CON PSA EN TRE 4 Y $10 \mathrm{ng} / \mathrm{ml}$.

\begin{tabular}{|l|c|c|c|c|c|c|c|}
\hline & & N & Media & DT & Mínimo & Máximo & $\begin{array}{c}\text { ANOVA } \\
\text { IN TERG RUPOS }\end{array}$ \\
\hline VO LUMEN & Total & 3440 & 6.81 & 1.59 & 4.00 & 9.99 & \\
\hline C.C. & HBP & 2268 & 42.58 & 20.92 & 3.53 & 320.00 & P<0,0001 \\
\hline & CANCER & 1013 & 29.04 & 14.77 & 3.20 & 129.60 & \\
\hline & PIN & 78 & 40.06 & 17.21 & 13.52 & 112.69 & \\
\hline & AG F & 71 & 36.71 & 18.03 & 10.89 & 111.25 & \\
\hline & Total & 3430 & 38.40 & 20.11 & 3.20 & 320.00 & \\
\hline
\end{tabular}


TABLA IV. DISTRIBUCIÓN DE AN ATO M ÍAS POR G RUPOS DE VO LUMEN EN PRIM ERA BIO PSIA.

\begin{tabular}{|c|c|c|c|c|c|}
\hline \multicolumn{6}{|c|}{ No } \\
\hline $\begin{array}{c}\text { Grupos } \\
\text { volumen(c.c.) }\end{array}$ & $\mathrm{n}$ & HBP & CANCER & PIN & AGF \\
\hline$<20$ & 875 & $\begin{array}{c}259 \\
(29,6)\end{array}$ & $\begin{array}{c}588 \\
(67,2)\end{array}$ & $\begin{array}{c}10 \\
(1,1)\end{array}$ & $\begin{array}{c}18 \\
(2,1)\end{array}$ \\
\hline $20-30$ & 1468 & $\begin{array}{c}665 \\
(45,3)\end{array}$ & $\begin{array}{c}743 \\
(50,6)\end{array}$ & $\begin{array}{c}24 \\
(1,6)\end{array}$ & $\begin{array}{c}36 \\
(2,5)\end{array}$ \\
\hline $30-40$ & 1397 & $\begin{array}{c}787 \\
(60,2)\end{array}$ & $\begin{array}{c}465 \\
(35,6)\end{array}$ & $\begin{array}{c}28 \\
(2,1)\end{array}$ & $\begin{array}{c}27 \\
(2,1)\end{array}$ \\
\hline $40-50$ & 858 & $\begin{array}{c}565 \\
(65,9)\end{array}$ & $\begin{array}{c}251 \\
(29,3)\end{array}$ & $\begin{array}{c}24 \\
(2,8)\end{array}$ & $\begin{array}{c}18 \\
(2,1)\end{array}$ \\
\hline$>50$ & 1464 & $\begin{array}{l}1112 \\
(76)\end{array}$ & $\begin{array}{c}289 \\
(19,7)\end{array}$ & $\begin{array}{c}36 \\
(2,5)\end{array}$ & $\begin{array}{c}27 \\
(1,8)\end{array}$ \\
\hline TOTAL & 5972 & $\begin{array}{c}3388 \\
(56,7)\end{array}$ & $\begin{array}{c}2336 \\
(39,1)\end{array}$ & $\begin{array}{c}122 \\
(2)\end{array}$ & $\begin{array}{l}126 \\
(2,1)\end{array}$ \\
\hline
\end{tabular}

tamaño mayor es la probabilidad de tacto sospechoso o ecografía anormal, resultando también esta asociación significativa $(p<0,0001)$.

Los parámetros basados en el PSA sérico en los diferentes grupos de volumen aparecen en la Tabla VI. La

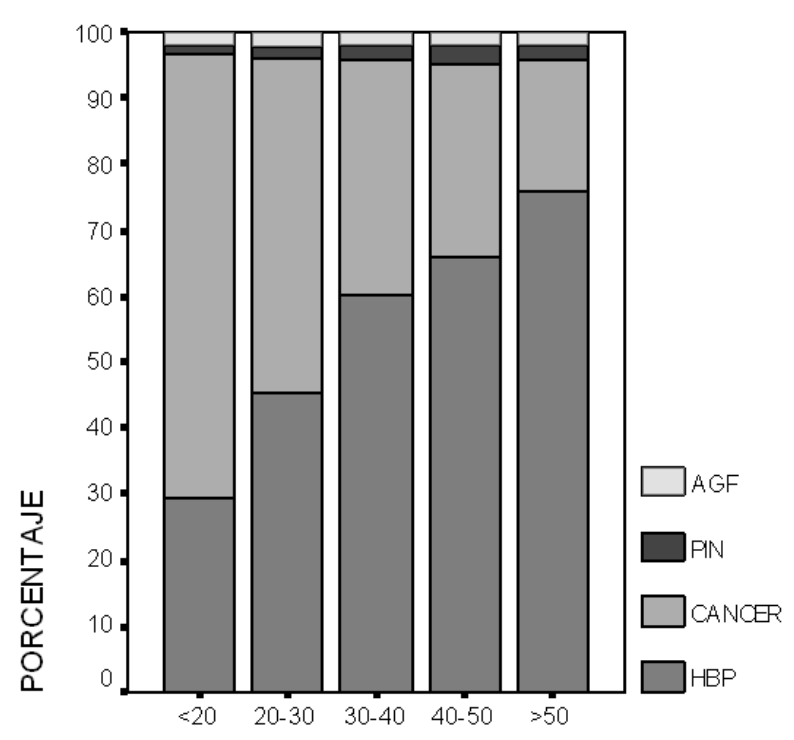

grupos de volumen

FIG URA 1. Histograma de distribución de la a natomía patológica en grupos de volumen en primera biopsia. diferencia en el PSA medio no es significativa lo que condiciona que la DPSA se reduzca en función del aumento de tamaño prostático $(p<0,0001)$. La relación inversa se observa con el PSA L T que aumenta desde 0,10 en las próstatas más pequeñas hasta 0,18 en las mayores de 50 c.c. $(p<0,0001)$.

En los pacientes con PSA entre 4 y $10 \mathrm{ng} / \mathrm{ml}$ se aprecia similar relación con una incidencia mayor del $50 \%$ de cánceres entre las próstatas de menos de 20 c.c. que disminuye con el incremento de volumen hasta resultar de un $8,9 \%$ en la mayores de 50 c.c. y $10,4 \%$ en las mayores de 40 c.c. (Tabla VII y Figura 2). La misma división del volumen se ha realizado en la segunda biopsia, encontrando unos resultados muy similares a los de pacientes con PSA entre 4 y 10 $\mathrm{ng} / \mathrm{ml}$ en la primera biopsia (Tabla VIII y Figura 3 ), salvo que en este grupo la proporción de próstatas de pequeño tamaño es menor con un 5,8\% menores de 20 c.c. frente al $14,7 \%$ en la primera biopsia 0 un $22,8 \%$ menores de 30 c.c. frente al $39,2 \%$ en la primera que concordaría con el hecho de que se hayan retirado gran parte de estas próstatas pequeñas al ser diagnosticadas con la biopsia inicial.

En las regresiones logísticas que incluyen PSA, DPSA, volumen fraccionados en categorías, el TR 
TABLA V. CARATERISTIC Q AS EC O G RÁFICAS Y DE TACTO RECTAL SEG ÚN GRUPOS DE VO LUM EN EN PRIMERA BIO PSIA.

\begin{tabular}{|l|c|c|c|c|c|c|}
\hline G rupos volumen(c.c.) & \multicolumn{2}{|c|}{ estructura homogénea } & \multicolumn{2}{c|}{$\begin{array}{c}\text { Nódulos hipoecoicos } \\
\text { No }\end{array}$} & \multicolumn{2}{c|}{ Tacto rectal } \\
& SI & N O & SI & N O & N O RM AL & PO SITIVO \\
\hline$<20$ & 644 & 217 & 375 & 456 & 530 & 310 \\
& $(74,8)$ & $(25,2)$ & $(45,1)$ & $(54,9)$ & $(63,1)$ & $(36,9)$ \\
\hline $20-30$ & 1226 & 212 & 426 & 993 & 1093 & 329 \\
& $(85,3)$ & $(14,7)$ & $(30)$ & $(70)$ & $(76,9)$ & $(23,1)$ \\
\hline $30-40$ & 1148 & 143 & 291 & 983 & 1051 & 219 \\
& $(88,9)$ & $(11,1)$ & $(22,8)$ & $(77,2)$ & $(82,8)$ & $(17,2)$ \\
\hline $40-50$ & 761 & 87 & 160 & 680 & 715 & 128 \\
& $(89,7)$ & $(10,3)$ & $(19)$ & $(81)$ & $(84,8)$ & $(15,2)$ \\
\hline$>50$ & 1331 & 116 & 206 & 1243 & 1259 & 168 \\
& $(92,0)$ & $(8)$ & $(14,2)$ & $(85,8)$ & $(88,2)$ & $(11,8)$ \\
\hline TOTAL & $\mathbf{5 1 1 0}$ & $\mathbf{7 7 5}$ & $\mathbf{1 4 5 8}$ & $\mathbf{4 3 5 5}$ & $\mathbf{4 6 4 8}$ & $\mathbf{1 1 5 4}$ \\
& $(\mathbf{8 6 , 8 )}$ & $\mathbf{( 1 3 , 2 )}$ & $\mathbf{( 2 5 , 1 )}$ & $\mathbf{( 7 4 , 9 )}$ & $\mathbf{( 8 , 1 )}$ & $\mathbf{( 1 9 , 9 )}$ \\
\hline
\end{tabular}

y la presencia de una ecografía sospechosa (nódulos hipoecoicos o próstata heterogénea) todos los factores resultaron significativos. EI PSA L/T se incluyó en un estudio de regresión aparte que incorporaba además las mismas categorías de DPSA y volumen, TR y ecografía. El PSA $L T$, aunque resultó significativo en el análisis univariante, fue excluido por el sistema en el análisis multivariante. La Tabla IX refleja los datos obtenidos. La combinación de pruebas consigue un área bajo la curva ROC de 0,84.

\section{DISCUSIÓN}

En este estudio el volumen prostático se ha revelado como un factor de gran importancia, tanto en el estudio univariante como en el multivariante, e independiente del PSA o sus índices. Babaian y cols (4) en 1996 establecieron unos rangos de PSA en función del volumen para indicar la biopsia aunque finalmente concluían que era preferible el empleo del punto de corte clásico de $4 \mathrm{ng} / \mathrm{ml}$. Con todo, Stamey en el comentario editorial a un artículo de Eskew y cols (6) dice que, en determinado sentido, el volumen podría considerarse como un marcador tumoral similar al
PSA puesto que cuanto mayor es una próstata mayor es la probabilidad de curación con prostatectomía radical.

El presente estudio evidencia que el volumen medio se incrementa en biopsias sucesivas, aunque a partir de la cuarta biopsia no resulta significativo; igualmente el volumen de las próstatas con tumor es menor que en aquellas en las que no se demostró malignidad pero esta diferencia sólo es significativa entre la primera y segunda biopsias. Catalona y cols (7) y Babaian y cols (8) encuentran una relación similar.

Este estudio pone de manifiesto que la incidencia de CP se modifica con el volumen prostático, variando desde el $67,2 \%$ en volúmenes inferiores a 20 c.c. al 19,7\% en próstatas mayores de 50c.c. En pacientes con PSA entre 4 y $10 \mathrm{ng} / \mathrm{ml}$ y en las segundas biopsias se mantiene esta tendencia incluso con una frecuencia inferior al $10 \%$ en glándulas superiores a 50 c.c. y cercana al mismo en glándulas entre 40 y 50 c.c. Presti (13) y Karakiewicz y cols (14) refieren una relación inversamente proporcional entre el volumen y la posibilidad de cáncer que es del 40 y $10 \%$ en 
TABLA VI. DESCRIPTIVOS PARA PSA, VO LUMEN, DPSA Y PSA LI TEN CADA GRUPO DE VO LUMEN EN LA PRIM ERA BIO PSIA.

\begin{tabular}{|c|c|c|c|c|c|c|c|c|}
\hline & $\begin{array}{c}\text { Grupo } \\
\text { volumen }\left(c_{.} c_{2}\right)\end{array}$ & N & Mediana & Media & DT & Mín. & Máx. & $\begin{array}{l}\text { Anova } \\
\text { intergr. }\end{array}$ \\
\hline PSA & $<20$ & 854 & 8.3 & 18.93 & 70.67 & .09 & 1368 & n.s. \\
\hline \multirow[t]{5}{*}{$(\mathrm{ng} / \mathrm{ml})$} & $20-30$ & 1445 & 7.8 & 18.23 & 71.70 & .50 & 1644 & \\
\hline & $30-40$ & 1283 & 7.63 & 23.76 & 139.61 & .07 & 3375 & \\
\hline & $40-50$ & 845 & 8.30 & 19.88 & 68.49 & 1.55 & 1049 & \\
\hline & $>50$ & 1443 & 9.73 & 29.26 & 181.96 & 2.19 & 4607 & \\
\hline & Total & 5870 & 8.35 & 22.49 & 122.79 & .07 & 4607 & \\
\hline VOLUMEN & $<20$ & 875 & 16.40 & 15.54 & 3.45 & 2.06 & 19.99 & $P<0.0001$ \\
\hline \multirow[t]{5}{*}{ (c.c) } & $20-30$ & 1468 & 24.98 & 24.95 & 2.78 & 20.05 & 29.95 & \\
\hline & $30-40$ & 1307 & 34.90 & 34.83 & 2.96 & 30.01 & 40.00 & \\
\hline & $40-50$ & 858 & 44.54 & 44.54 & 2.78 & 40.18 & 49.92 & \\
\hline & $>50$ & 1464 & 63.88 & 73.99 & 126.89 & 50.27 & 4837.7 & \\
\hline & Total & 5972 & 34.68 & 40.57 & 66.23 & 2.06 & 4837.7 & \\
\hline DPSA & $<20$ & 854 & 0.52 & 1.25 & 4.18 & .01 & 73.94 & $P<0.0001$ \\
\hline \multirow[t]{5}{*}{ (ng/ ml/ c.c.) } & $20-30$ & 1445 & 0.31 & .75 & 3.22 & .02 & 81.19 & \\
\hline & $30-40$ & 1283 & 0.22 & .67 & 3.79 & .00 & 88.76 & \\
\hline & $40-50$ & 845 & 0.18 & .53 & 2.98 & .03 & 73.55 & \\
\hline & $>50$ & 1443 & 0.14 & .46 & 3.14 & .03 & 80.67 & \\
\hline & Total & 5870 & 0.23 & .70 & 3.46 & .00 & 88.76 & \\
\hline \multirow[t]{6}{*}{ PSAL/T } & $<20$ & 311 & 0.09 & .10 & .05 & .00 & .41 & $P<0.0001$ \\
\hline & $20-30$ & 586 & 0.11 & .12 & .06 & .02 & .60 & \\
\hline & $30-40$ & 545 & 0.14 & .15 & .07 & .00 & .55 & \\
\hline & $40-50$ & 342 & 0.14 & .15 & .06 & .00 & .44 & \\
\hline & $>50$ & 553 & 0.17 & .18 & .08 & .01 & .52 & \\
\hline & Total & 2337 & 0.13 & .14 & .07 & .00 & .60 & \\
\hline
\end{tabular}

próstatas menores de 20 y mayores de 80 c.c. respectivamente. Roehl y cols $(15,16)$ encuentran un $27-44 \%$ de tumores con volúmenes por debajo de 30 c.c. Incluso en las series de biopsias ampliadas es general que la mayoría de los autores refieran menor incidencia de CP a mayor volumen prostático (17-20).

La diferencia entre los porcentajes de CP según el volumen prostático sugiere que hay algo erróneo en el planteamiento de la biopsia. No parece lógico que más del $50 \%$ de pacientes con PSA entre 4 y $10 \mathrm{ng} / \mathrm{ml}$ y próstata menor de $20 \mathrm{cc}$ tengan un tumor en la biopsia mientras que menos de un $9 \%$ lo tengan si su volumen es mayor de $50 \mathrm{cc}$. Pueden plantearse dos explicaciones: 0 el número de muestras es insuficiente en próstatas mayores o realmente estas tienen menos cáncer y el PSA deriva de la HPB que incrementa su tamaño. La tendencia actual es a incrementar el número de muestras en la BTE dado que numerosos autores consideran insuficiente la biopsia por sextantes para el diagnóstico del cáncer en próstatas de mayor volumen. A sí Remzi y cols (9) y Djavan y cols (10) refieren un mayor volumen de las glándulas con tumor que se diagnosticaron en la segunda biopsia 
TABLA VII. DISTRIBUCIÓ N DE AN ATO M ÍAS PATO LÓ G ICAS PO R G RUPO S DE VO LUM EN EN PACIEN TES DE PRIM ERA BIO PSIA CON PSA $4-10 \mathrm{ng} / \mathrm{ml}$.

\begin{tabular}{|c|c|c|c|c|c|}
\hline \multicolumn{6}{|c|}{ No } \\
\hline $\begin{array}{c}\text { Grupos } \\
\text { volumen(c.c.) }\end{array}$ & $\mathrm{n}$ & HBP & CAN CER & PIN & AGF \\
\hline$<20$ & 251 & $\begin{array}{c}111 \\
(44.2)\end{array}$ & $\begin{array}{c}131 \\
(52.2)\end{array}$ & $\begin{array}{c}4 \\
(1.6)\end{array}$ & $\begin{array}{c}5 \\
(2.0)\end{array}$ \\
\hline $20-30$ & 612 & $\begin{array}{c}414 \\
(67.6) \\
\end{array}$ & $\begin{array}{c}161 \\
(26.3) \\
\end{array}$ & $\begin{array}{c}17 \\
(2.8) \\
\end{array}$ & $\begin{array}{c}20 \\
(3.3) \\
\end{array}$ \\
\hline $30-40$ & 648 & $\begin{array}{c}514 \\
(79.3)\end{array}$ & $\begin{array}{c}107 \\
(16.5)\end{array}$ & $\begin{array}{c}15 \\
(2.3)\end{array}$ & $\begin{array}{c}12 \\
(1.9)\end{array}$ \\
\hline $40-50$ & 431 & $\begin{array}{c}352 \\
(81.7)\end{array}$ & $\begin{array}{c}55 \\
(12.8)\end{array}$ & $\begin{array}{c}17 \\
(3.9)\end{array}$ & $\begin{array}{c}7 \\
(1.6)\end{array}$ \\
\hline$>50$ & 653 & $\begin{array}{c}568 \\
(87.0)\end{array}$ & $\begin{array}{c}58 \\
(8.9)\end{array}$ & $\begin{array}{c}14 \\
(2.1)\end{array}$ & $\begin{array}{c}13 \\
(2.0)\end{array}$ \\
\hline TOTAL & 2595 & $\begin{array}{l}1959 \\
(75.5)\end{array}$ & $\begin{array}{c}512 \\
(19.7)\end{array}$ & $\begin{array}{c}67 \\
(2.6)\end{array}$ & $\begin{array}{c}57 \\
(2.2)\end{array}$ \\
\hline
\end{tabular}

frente a las que se diagnosticaron en la primera, aconsejando rebiopsiar a pacientes con un volumen total mayor de $45 \mathrm{cc}$. En este estudio la media de volumen

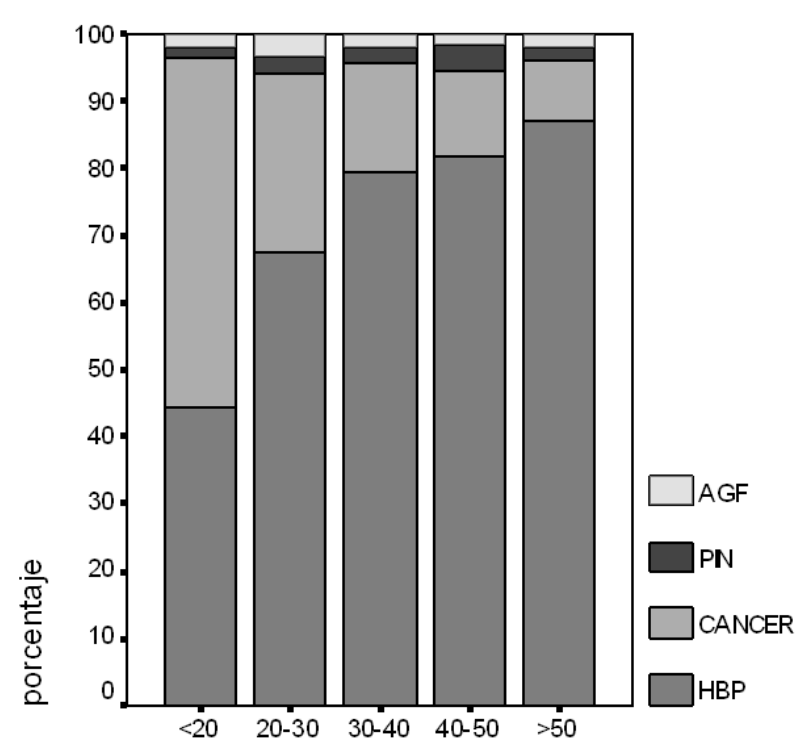

grupos de volumen

FIG URA 2. Histograma de distribución de la anatomía patológica en grupos de volumen en pacientes con PSA entre 4 y $10 \mathrm{ng} / \mathrm{ml}$. en pacientes con $\mathrm{CP}$ en las cuatro primeras biopsias es de $27,2,30,5,35$ y 34 cc respectivamente, es decir, que aunque el volumen aumenta en las tres primeras,

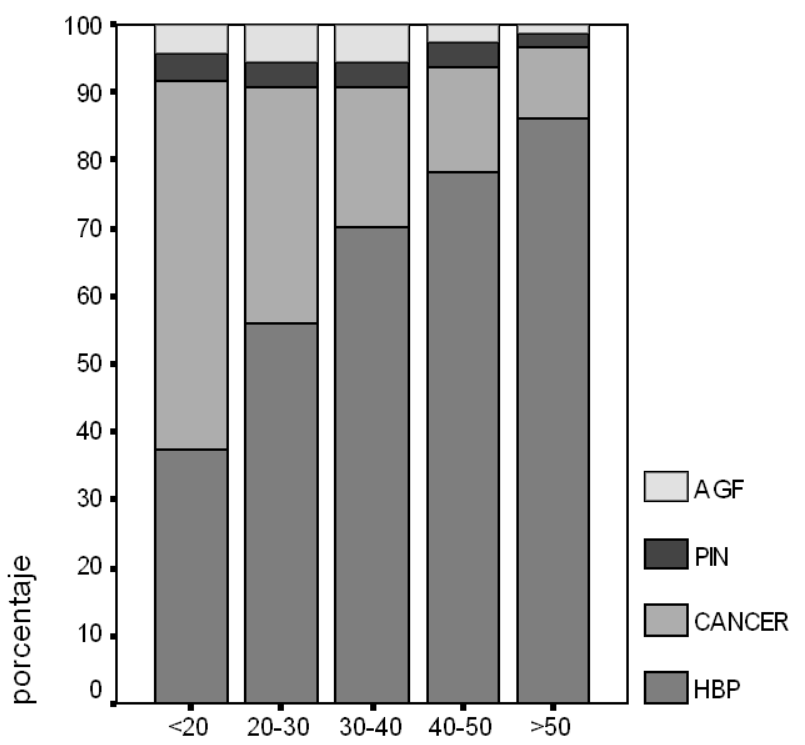

grupos por volumen

FIG URA 3. Histograma con distribución de a natomías según grupos de volumen en la segunda biopsia. 
después de 4 biopsias siguen diagnosticándose cánceres en próstatas relativamente pequeñas y no en las de mayor tamaño. Basillote y cols (11) refieren que el porcentaje de tumores no diagnosticados en la primera biopsia es el doble en próstatas mayores de 37,5 c.c. que en las menores. Por el contrario Ung y cols (12) sin embargo aunque refieren una disminución del porcentaje de cánceres con el incremento del tamaño, no encuentra que el aumento de muestras aumente la tasa de detección, concluyendo que el aumento de PSA en próstatas grandes puede deberse a otras fuentes no tumorales y que en estos quizás debería elevarse el nivel de PSA a partir del cual biopsiar. Distintos autores han demostrado un incremento diagnóstico de las biopsias ampliadas frente a la sextante (17-19) pero si se analizan estas series se observa que la base de comparación es la biopsia sextante clásica según describieron Hodge y cols (21) donde las muestras se obtienen a mitad de camino entre la línea media y el borde externo prostático con lo que el cilindro de tejido incluye tanto zona periférica como adenoma con un rendimiento diagnóstico menor. En nuestro grupo, obteniendo desde el principio muestras de los cuernos laterales prostáticos el porcentaje diagnóstico se aproxima a muchas series con 8 ó más cilin$\operatorname{dros}(19,22,23)$.
El aumento del tamaño prostático es debido fundamentalmente al crecimiento de una HPB mientras que la zona periférica mantiene un volumen similar por tanto, si los cilindros de tejido proceden puramente de la zona periférica, el porcentaje de tejido periférico obtenido será similar en una glándula de $20 \mathrm{cc}$ que en una de $80 \mathrm{cc}$, reconociendo que en próstatas con gran adenoma puede ser difícil la obtención por vía transrectal de tejido exclusivamente periférico. De ahí que pensemos que es más importante la localización que el número de muestras.

A poyando la idea de la relación del PSA con el volumen, Stamey y cols (24) tras revisar 1317 prostatectomías radicales consecutivas en periodos de 4-5 años encuentran que en los últimos 5 años el PSA sólo ha presentado relación con el volumen de la glándula y con ningún otro parámetro histopatológico del cáncer, concluyendo que en los últimos años el PSA sólo refleja la HPB presente en la próstata.

Por otro lado que más de la mitad de pacientes con próstata de tamaño normal presenten cáncer parece indicar que estamos biopsiando tarde a este grupo de riesgo. Si consideramos que Catalona y cols (25) encuentran respecto al estadio

TABLA VIII. DISTRIBUCIÓN DE AN ATO M ÍAS POR GRUPO S DE VO LUM EN EN LA SEG UN DA BIO PSIA.

\begin{tabular}{|c|c|c|c|c|c|}
\hline \multicolumn{7}{|c|}{ No } \\
\begin{tabular}{|c|c|c|c|c|} 
Grupos \\
volumen(c.c.)
\end{tabular} & $\mathbf{n}$ & HBP & CANCER & PIN & AG F \\
\hline$<20$ & 48 & 18 & 26 & 2 & 2 \\
& & $(37.5)$ & $(54.2)$ & $(4.2)$ & $(4.2)$ \\
\hline $20-30$ & 139 & 78 & 48 & 5 & 8 \\
& & $(56.1)$ & $(34.5)$ & $(3.6)$ & $(5.8)$ \\
\hline $30-40$ & 174 & 122 & 36 & 6 & 10 \\
& & $(70.1)$ & $(20.7)$ & $(3.4)$ & $(5.7)$ \\
\hline $40-50$ & 146 & 114 & 23 & 5 & 4 \\
& & $(78.1)$ & $(15.8)$ & $(3.4)$ & $(2.7)$ \\
\hline$>50$ & 315 & 271 & 34 & 6 & 4 \\
& & $(86.0)$ & $(10.8)$ & $(1.9)$ & $(1.3)$ \\
\hline TOTAL & $\mathbf{8 2 2}$ & $\mathbf{6 0 3}$ & $\mathbf{1 6 7}$ & $\mathbf{2 4}$ & $\mathbf{2 8}$ \\
& & $\mathbf{( 7 3 . 4 )}$ & $\mathbf{( 2 0 . 3 )}$ & $\mathbf{( 2 . 9 )}$ & $\mathbf{( 3 . 4 )}$ \\
\hline
\end{tabular}


patológico que con una DPSA mayor de 0,15 $\mathrm{ng} / \mathrm{ml} / \mathrm{c}$.c. sólo el $36 \%$ de pacientes tienen características favorables en la anatomía patológica definitiva y aproximadamente un $33 \%$ con un PSA L/T menor de 0,15 , mientras que con DPSA menor de $0,15 \mathrm{ng} / \mathrm{ml} / \mathrm{c}$. c. y PSA L/T mayor de 0,15 el $75 \%$ de pacientes tienen tumores de buen pronóstico (organoconfinados, Gleason $<7$, menos del $10 \%$ de afectación glandular), significaría que en próstatas de pequeño volumen estamos diagnosticando tumores agresivos y de peor pronóstico con los puntos de corte estándar de $4 \mathrm{ng} / \mathrm{ml}$, ya que la mayoría presentan DPSA más altas y PSA L/T más bajos.

Estos datos parecen querer indicar la necesidad de abandonar los dinteles estrictos de PSA para ajustarlos individualmente, siendo el volumen uno de los factores primordiales para ello. Tomando como referencia que pacientes con DPSA de $0,14 \mathrm{ng} / \mathrm{ml} / \mathrm{cc}$ presentan una incidencia de $C P$ del $15 \%$ se calcula que para una rentabilidad similar sería necesario bajar a un PSA de $2,8 \mathrm{ng} / \mathrm{ml}$ en una próstata de 20 cc para obtener la misma DPSA y $4,2 \mathrm{ng} / \mathrm{ml}$ si la prós- tata fuera de $30 \mathrm{cc}$. Contrariamente en las próstatas más voluminosas la rentabilidad es muy baja con los puntos de corte actuales por lo que parecería más lógico no biopsiar por debajo de $10 \mathrm{ng} / \mathrm{ml}$ con volúmenes superiores a $50 \mathrm{cc}$. Puede argumentarse que un $8,9 \%$ de tumores justifica la biopsia, pero si pensamos que esta cifra no está muy lejos de lo que encuentran Thompson y cols (26) del $6,6 \%$ en pacientes con PSA menor de $0,5 \mathrm{ng} / \mathrm{ml} 0$ el $10,1 \%$ en aquellos entre 0,6 y $1 \mathrm{ng} / \mathrm{ml}$ estaríamos justificando el biopsiar a todo varón independientemente del PSA.

\section{CONCLUSIONES}

Los puntos de corte estándar de PSA no son adecuados para un correcto diagnóstico de CP mediante BTE. El volumen (HPB) influye de manera determinante en sus cifras y en la rentabilidad de la prueba por lo que es preciso disminuir el dintel en próstatas de menor tamaño e incrementarlo en las mayores, no justificándose la biopsia en glándulas por encima de $50 \mathrm{cc}$ con PSA inferior a $10 \mathrm{ng} / \mathrm{ml}$.

TABLA IX. REG RESIÓ N M ULTIVARIAN TE PATA PSA, DPSA, VO LUM EN, TR Y ETR.

\begin{tabular}{|c|c|c|c|c|c|}
\hline \multirow[t]{2}{*}{ Parametro } & \multirow{2}{*}{$\chi^{2} W$ ald } & \multirow{2}{*}{$\operatorname{Pr}>\chi^{2}$} & \multicolumn{3}{|c|}{0 dds ratio estimado } \\
\hline & & & Punto estimado & Intervalo c & a al $95 \%$ \\
\hline PSA $6-10$ & 0.1575 & 0.6915 & 1.042 & 0.850 & 1.278 \\
\hline PSA $10-33$ & 4.7555 & 0.0292 & 1.350 & 1.031 & 1.768 \\
\hline PSA $>33$ & 39.4028 & $<.0001$ & 3.915 & 2.556 & 5.994 \\
\hline DPSA $0.13-0.18$ & 2.2377 & 0.1347 & 1.245 & 0.934 & 1.659 \\
\hline DPSA $0.18-0.26$ & 14.4893 & 0.0001 & 1.758 & 1.315 & 2.351 \\
\hline DPSA $\geq 3 \mathrm{~d} 0.26$ & 42.9228 & $<.0001$ & 3.124 & 2.222 & 4.392 \\
\hline Volumen $20-30$ & 11.4498 & 0.0007 & 0.692 & 0.559 & 0.856 \\
\hline Volumen $30-40$ & 37.0644 & $<.0001$ & 0.470 & 0.368 & 0.599 \\
\hline Volumen $40-50$ & 40.9086 & $<.0001$ & 0.391 & 0.293 & 0.521 \\
\hline Volumen >50 & 73.1645 & $<.0001$ & 0.256 & 0.188 & 0.350 \\
\hline TR sospechoso & 170.9448 & $<.0001$ & 3.743 & 3.071 & 4.563 \\
\hline ETR sospechosa & 228.8563 & $<.0001$ & 3.535 & 3.001 & 4.163 \\
\hline
\end{tabular}

likelihood ratio: $\chi^{2} 2263.67 \mathrm{GL}=12 p<0,0001$

PSA (ng/ ml); DPSA (ng/ml/ c.c.); Volumen (c.c.) 


\section{BIBUOGRAFIA y LECTURAS RECOMENDADAS (*lectura de interés y $* *$ lectura fundamental)}

1. MOROTE, J.; RUIBAL, A.; PALOU, J.: "Evaluation of specific antigen and prostatic acid phosphatase specificity. Study of false values". Int. J. Biol. Markers, 1: 141, 1986.

2. ARCANGELI, C.G.; ORNSTEIN, D.K.; KEETCH, D.W. y cols.: "Prostate specific antigen as a screening test for prostate cancer: the United States experience". Urol. Clin. North Am., 24: 299, 1997.

*3. BENSON, M.C.; WHANG, I.S.; PANTUCK, A. y cols.: "Prostate specific antigen density: a means of distinguishing benign prostatic hypertrophy and prostate cancer". J. Urol., 147: 815, 1992.

*4. BABAIAN, R.J.; KOJIMA, M.; RAMIREZ, E.I. y cols.: "Comparative analysis of prostate specific antigen and its indexes in the detection of prostate cancer". J. Urol., 156: 432, 1996.

5. TERRIS, M.K.; STAMEY, T.A.: "Determination of prostate volume by transrectal ultrasound". J. Urol., 145: 984, 1991

6. ESKEW, L.A.; BARE, R.L.; McCULLOUGH, D.L.: "Systematic 5 region prostate biopsy is superior to sextant method for diagnosing carcinoma of the prostate". J. Urol., 157: 199, 1997.

7. CATALONA, W.J.; PARTIN, A.W.; FINLAY, J.A. y cols.: "Use of percentage of free prostate-specific antigen to identify men at high risk of prostate cancer when PSA levels are 2.51 to $4 \mathrm{ng} / \mathrm{ml}$ and digital rectal examination is not suspicious for prostate cancer: an alternative model". Urology, 54: 220, 1999.

8. BABAIAN, R.J.; JOHNSTON, D.A.; NACCARATO, W. y cols.: "The incidence of prostate cancer in a screening population with a serum prostate specific antigen between 2.5 and $4.0 \mathrm{ng} / \mathrm{ml}$ : relation to biopsy strategy". J. Urol., 165: 757, 2001.

*9. REMZI, M. ; DJAVAN, B.; WAMMACK, R. y cols. : "Can total and transition zone volume of the prostate determine whether to perform a repeat biopsy?". Urology, 61: 161, 2003.

*10. DJAVAN, B.; ZLOTTA, A.R.; EKANE, S. y cols.: "Is one set of sextant biopsies enough to rule out prostate cancer? Influence of transition and total prostate volumes on prostate cancer yield". Eur. Urol., 38: 218, 2000.

*11. BASILLOTE, J.B.; ARMENAKAS, N.A.; HOCHBERG, D.A. y cols.: "Influence of prostate volume in the detection of prostate cancer". Urology, 61: 167, 2003.

*12. UNG, J.O.; SAN FRANCISCO, I.F.; REGAN, M.M. y cols.: "The relationship of prostate gland volume to extended needle biopsy on prostate cancer detection". J. Urol., 169: 130, 2003

*13. PRESTI, J.C.Jr.: "Prostate biopsy: how many cores are enough?”. Urol. Oncol., 21: 135, 2003.

14. KARAKIEWICZ, P.I.; BAZINET, M.; APRIKIAN, A.G. y cols.: "Outcome of sextant biopsy according to gland volume". Urology, 49: 55, 1997.

15. ROEHL, K.A.; ANTENOR, J.A.; CATALONA, W.J.: "Serial biopsy results in prostate cancer screening study". J. Urol., 167: 2435, 2002.

16. ROEHL, K.A.; ANTENOR, J.A.; CATALONA, W.J.: "Robustness of free prostate specific antigen measurements to reduce unnecessary biopsies in the 2.6 to 4.0 ng/ml range". J. Urol., 168: 922, 2002.

*17. BABAIAN, R.J.; TOI, A.; KAMOI, K. y cols.: "Comparative analysis of sextant and an extended 11core multisite directed biopsy strategy". J. Urol., 163: $152,2000$.

18. LEVINE, M.A.; ITTMAN, M.; MELAMED, J. y cols.: "Two consecutive sets of transrectal ultrasound guided sextant biopsies of the prostate for the detection of prostate cancer". J. Urol., 159: 471, 1998.

*19. RAVERY, V.; GOLDBLATT, L.; ROYER, B. y cols.: "Extensive biopsy protocol improves the detection rate of prostate cancer". J. Urol., 164: 393, 2000.

*20. NAUGHTON, C.K. ; MILLER, D.C. ; MAGER, D.E. y cols.: "A prospective randomized trial comparing 6 versus 12 prostate biopsy cores: impact on cancer detection". J. Urol., 164: 388, 2000.

21. HODGE, K.K.; McNEAL, J.E.; TERRIS, M.K. y cols.: "Random systematic versus directed ultrasound guided transrectal core biopsies of the prostate". J. Urol., 142: 71, 1989.

**22. PRESTI, J.C.Jr.; CHANG, J.J.; BHARGAVA, V. y cols.: "The optimal systematic prostate biopsy scheme should include 8 rather than 6 biopsies: results of a prospective clinical trial". J. Urol., 163: 163, 2000.

*23. GORE, J.L.; SHARIAT, S.F.; MILES, B.J. y cols.: "Optimal combinations of systematic sextant and laterally directed biopsies for the detection of prostate cancer". J. Urol., 165: 1554, 2001.

**24. STAMEY, T.A.; CALDWELL, M.; McNEAL, J.E. y cols.: "The prostate specific antigen era in the United States is over for prostate cancer: what happened in the last 20 years?". J. Urol., 172: 1297, 2004.

*25. CATALONA, W.J.; SOUTHWICK, P.C.; SLAWIN, K.M. y cols.: "Comparison of percent free PSA, PSA density, and age-specific PSA cutoffs for prostate cancer detection and staging". Urology, 56: 255, 2000.

**26. THOMPSON, I.M.; PAULER, D.K.; GOODMAN, P.J. y cols.: "Prevalence of prostate cancer among men with a prostate-specific antigen level $\leq 4.0 \mathrm{ng}$ per mililiter". N. Engl. J. Med., 350: 2239, 2004. 